$$
\text { CONF-900756-. } 48
$$

UCRL- JC- 104995

PREPRINT

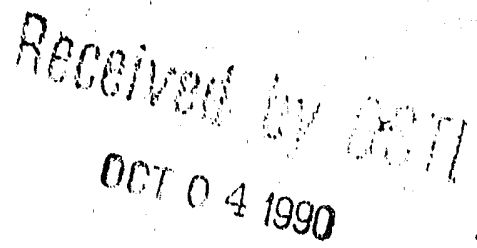

\title{
TEST RESULTS OF A PROTOTYPE DIELECTRIC MICROCALORIMETER
}

T.E. Pfafman, E. Silver, and S. Labov Lawrence Livermore National Laboratory

J. Beeman, F. Goulding, W. Hansen,

D. Landis, and N. Madden Lawrence Berkeley Laboratory

SPIE's 1990 International Symposium on Optical and Optoelectronic Applied Science and Engineering San Diego, CA, 7-13 July 1990

August 13, 1990

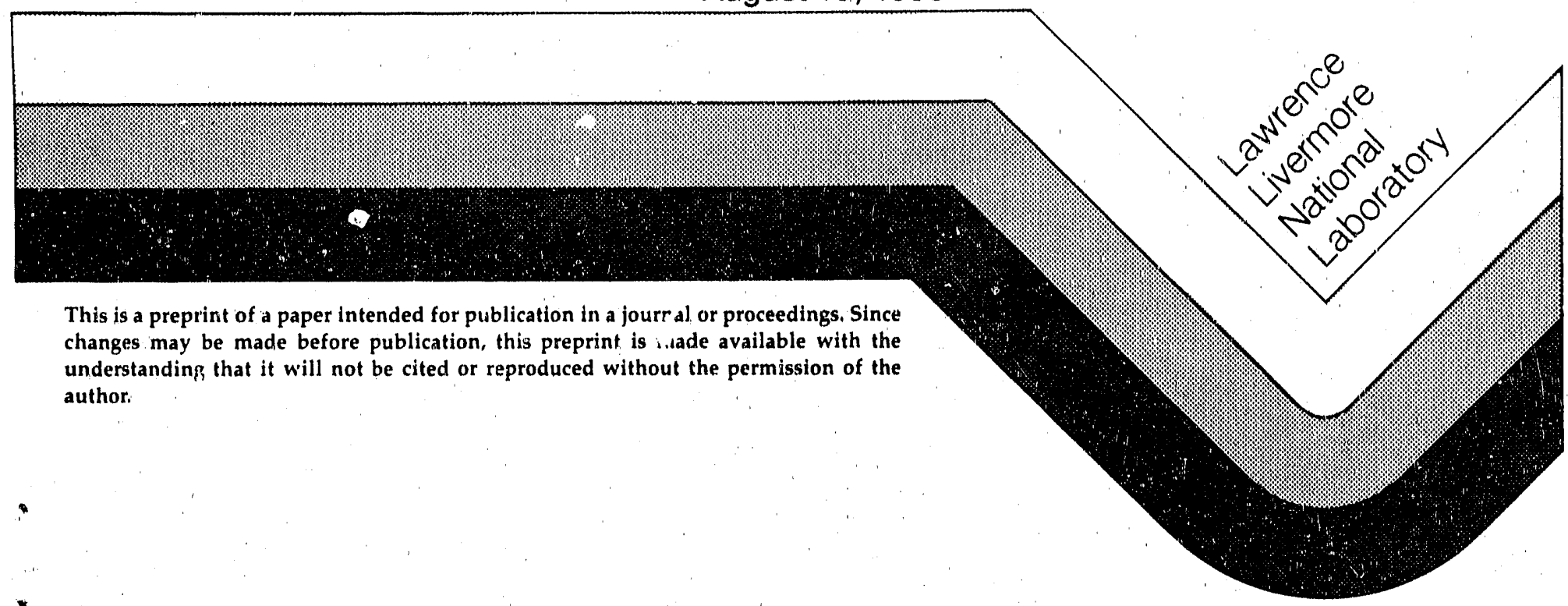


DISCIAIMER

This documeit was prepared as an account of work sponsored by an agency of the United Stales Government. Neither the Inited States Government nor the University of California nor any of their emplnyees, makes any warranty, express or implied, or assumes any legal liability or responsibility for the accuracy, completeness, or usefulness of any information, apparatus, product, or precess disclosed, or represents that its use would not infringe privately owned rights. Reference herein to any specific commercial products, process, or service by trade name, trademark, manufacturer, or otherwise, doe's not necessarily constitute or imply its endorsement, recommendation, or favoring by the United States Government or the University of California. The views and opinions of authors expressed herein do not necessarily state or reflect those of the United States Government or the University of California, and shall not be used for advertising or product endorsement purposes. 


\author{
T.E. Pfafman ', E. Silver, and S. Labov \\ Laboratory for Experimental Astrophysics \\ Lawrence Livermore National Laboratory
}

J. Beeman, F. Goulding, W. Hansen, D. Landis, and N. Madden

Lawrence Berkeley Laboratory

\begin{abstract}
The initial development work on a dielectric microcalorimeter is presented. It focuses on the dielectric properties of the ferroelectric material $\mathrm{KTa}_{1-\mathrm{x}} \mathrm{Nb}_{\mathrm{x}} \mathrm{O}_{3}(\mathrm{KTN})$. Measurements of the temperature dependent dielectric constant are given together with the first alpha particle detection results from a prototype composite microcalorimeter operating at $1.3 \mathrm{~K}$. A non-thermal mechanism for detecting $6 \mathrm{MeV}$ alpha particles in a monolithic KTN sample is also reported.
\end{abstract}

\title{
1. INTRODUCTION
}

Cryogenic calorimeters are being developed to meet the needs of scientists working in a wide variety of scientific and industrial settings. X-ray astronomers require spectrometers with high resolving power, broad band spectral coverage, and close to $100 \%$ efficiency at energies extending above $1 \mathrm{keV}$. Particle physicists interested in neutrino astrophysics and Dark Matter searches might also use these detectors because they offer a lower detection threshold than traditional ionization detectors. Studies of impurities in laboratory plasmas should also benefit from the reduced measurement complexity that a single broad band detector will provide, while chemical analysis using these low temperature detectors will be simpler and less time consuming than traditional diffraction techniques. Recert research on calorimetric detectors in this program and elsewhere indicates their promise as spectrometers.

In a parallel program, we are developing resistive calorimeters that operate at $0.1 \mathrm{~K}$ with an energy resolution as low as $19 \mathrm{eV}{ }^{1}$ The resolution of such calorimeters is limited because resistors generate Johnson noise. We are therefore investigating non-resistive methods to measure the small temperature changes in $\mathrm{x}$-ray absorbing materials. In principle, a ferroelectric temperature sensor that takes advantage of the change in dielectric constant with temperature can be made to behave like an excellent capacitor, thereby eliminating the Johnson noise and heat dissipation. Our early calculations showed that such a device could, in principle, be operated at $300 \mathrm{mK}$ with a resolution that is comparable to that of a resistive device operated below $100 \mathrm{mK}^{2}$ This would be a big advantage for $x$-ray astronomy payloads because a 300 $\mathrm{mK}$ operating temperature can be achieved with a ${ }^{3} \mathrm{He}$ refrigerator that is simpler, less expensive and stronger while providing more cooling power than a refrigerator required to achieve $100 \mathrm{mK}$.

\section{DIELECTRIC CALORIMETERS}

\subsection{General calorimetry}

A calorimeter is defined as a device that has a measurable temperature change when it absorbs a quantum of energy. When a quantum of energy $E$ is absorbed by a calorimeter with heat capacity $C_{\mathrm{v}}$ a temperature transient occurs of the form $\Delta T(t)=T(t)-T_{b}=T_{o} \exp ^{-t / \tau}$. Here $t$ is the time, $T_{b}$ is the temperature of the cold stage, $T_{o}=E / C_{v}$, and $\tau=C_{\mathrm{v}} / G$, where $G$ is the thermal conductivity of the link with the cold stage. Resistive calorimeters make use of the strong temperature dependence of resistance in doped semiconductor crystals at low temperatures to convert this

${ }^{1}$ T.E.Pfafman is also a graduate student in the Physics Department at the University of California at

Berkeley. 
temperature change into a useful voltage signal. Unfortunately, the electronic conversion introduces Johnson noise from the resistive elements of the circuit, JFET noise associated with the preamplifier, and mechanically-induced microphonic noise. These noise terms add in quadrature to the "phonon noise" which is a term used to describe the intrinsic thermal fluctuations in the device resulting from the random exchange of phonons between the cold stage and the thermometer. If the other noise terms mentioned above could be eliminated the phonon noise would be the only limitation. Since the phonon noise is usually dominated by Johnson noise at high frequencies in a resistive calorimeter, a dielectric calorimeter should permit high frequency operation with improved signal to noise.

\subsection{The dielectric microcalorimeter}

Dielectric microcalorimeters take advantage of the temperature dependence of the dielectric constant in ferroelectric materials to convert temperature perturbations into measurable voltage signals. The electronic method used to read out the signal is similar to that used in conventional ionization photon spectrometers ${ }^{3}$ and is shown in Figure 1. A ferroelectric capacitor, $C_{C}$, is connected to the input of a charge sensitive preamplifier. When the quantum of energy is absorbed, the resulting temperature change alters the dielectric constant, $\varepsilon$, of the ferroelectric. With a constant voltage bias applied across $C_{C}$, the charge on the capacitor will change by an amount, $\Delta Q$,

$$
\Delta Q=C_{\mathrm{c}} V_{\text {bias }} \frac{1}{\varepsilon} \frac{\mathrm{d} \varepsilon}{\mathrm{d} T} \Delta T(t) .
$$

The current pulse, $I_{C}=\mathrm{d} Q / \mathrm{d} t$, is integrated across the feedback capacitor, $C_{\mathrm{f}}$, to produce a voltage, $\Delta \mathrm{V}_{\text {out }}$,

$$
V_{\text {out }}(t)=\int_{0}^{t} \frac{I_{\mathrm{c}} \mathrm{d} t}{C_{\mathrm{f}}}=\frac{C_{\mathrm{c}} V_{\text {bias }}}{C_{\mathrm{f}}} \frac{1}{\varepsilon} \frac{\mathrm{d} \varepsilon}{\mathrm{d} T} \frac{E}{C_{\mathrm{v}}} \mathrm{e}^{-t / \tau} .
$$

A detailed discussion of the noise is presented in Silver et. al. ${ }^{1}$, and will not be reproduced here. Figure 2 shows the power spectra of the noise sources in a dielectric calorimeter when used with a charge sensitive preamplifier. In relative terms the Johnson noise is essentially zero above $10 \mathrm{~Hz}$, leaving the phonon noise term as the dominate noise source over a wide range of frequencies. Figure 2 also shows the bandpass efficiency for a post amplifying filter with a peaking time of $25 \mu \mathrm{s}$. If the signal has a fast enough risetime to allow operating with short filter time constants which strongly attenuate low frequencies, then a dielectric microcalorimeter operating at $0.3 \mathrm{~K}$ could, in theory, give a resolution $\left(\Delta E_{\mathrm{FWHM}}\right)$ of less than $4 \mathrm{eV}$, where a comparable resistive calorimeter operating at $0.1 \mathrm{~K}$ would achieve only $10 \mathrm{eV}^{4}$

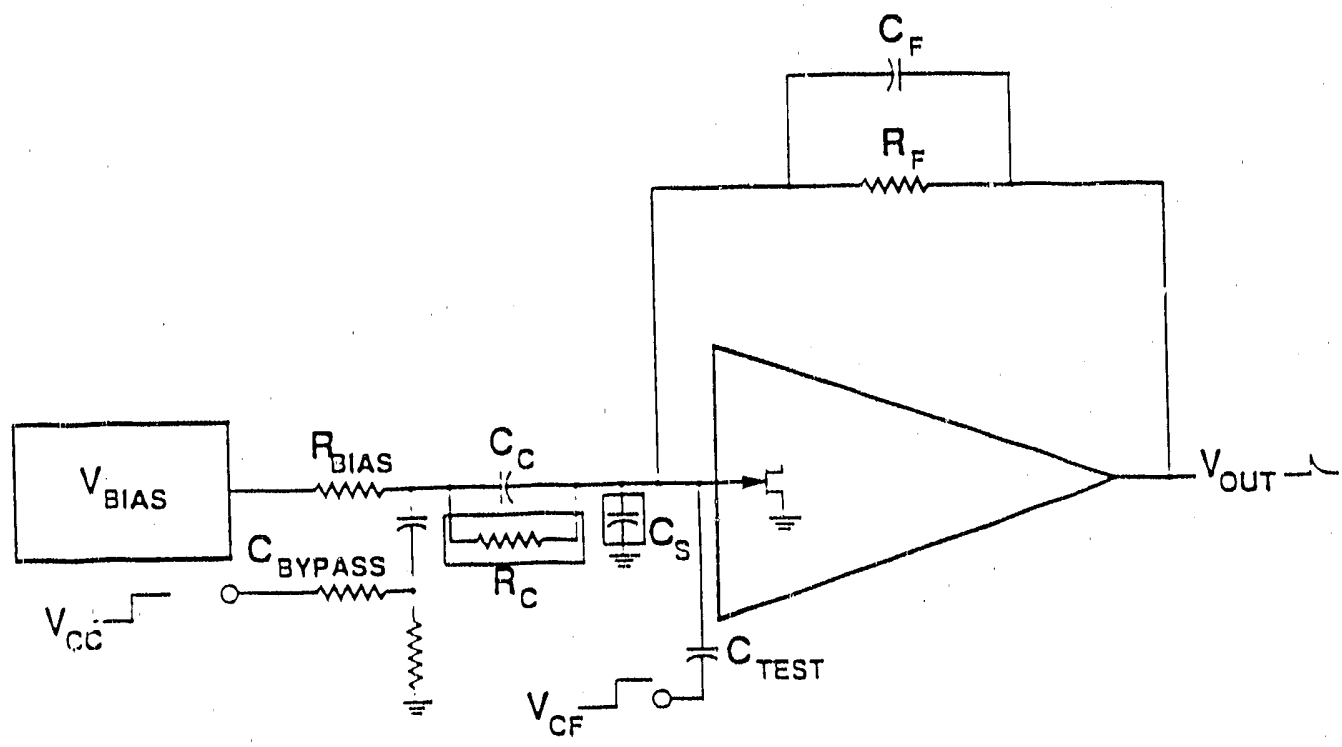

Figure 1. The schematic of the charge-sensitive preamplifier configuration used with the dielectric calorimeter. 


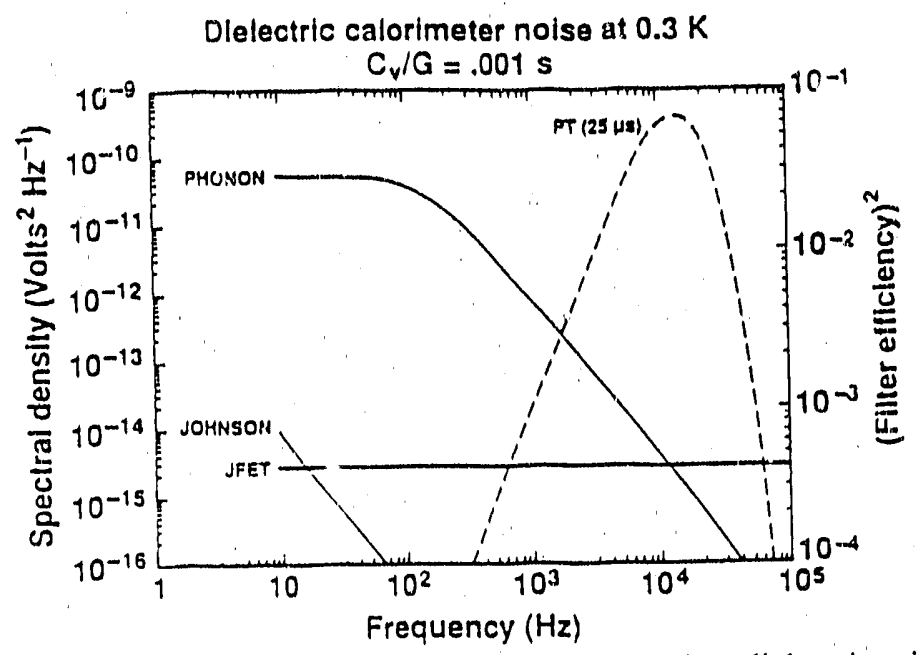

Figure 2. The frequency dependence of the noise components in a dielectric miciocalorimeter. The solid curves are the power spectra of the phonon, Johnson, and JFET noise. The dashed curve is the square of the post amplifier bandpass efficiency for a filter with a peaking time of $25 \mu \mathrm{s}$.

\section{MEASUREMENTS OF THERMAL PROPERTIES}

\subsection{Dielectric materials and sample preparation}

We present various sets of measurements of four different size capacitors. Two were used directly as monolithic microcalorimeters and two served as the thermal sensors in a composite configuration, where they were attached to a sapphire absorber. The samples are of the ferroelectric material $\mathrm{KTa}_{1-\mathrm{x}} \mathrm{Nb}_{\mathrm{x}} \mathrm{O}_{3}(\mathrm{KTN})$ with $\mathrm{x}=0.012$, which was supplied by D. Rytz. ${ }^{5}$. This material has a very large value for $\varepsilon$ and $\frac{1}{\varepsilon} \frac{d \varepsilon}{d T}$ below $4 \mathrm{~K}$, as can be seen in Figure 3 . This material potentially has a small heat capacity 6 and we have measured a large bulk resistivity, for our samples; the resistance was typically $R_{c} \geq 3 \times 10^{15} \Omega$. The large dielectric constant means that we can choose the geometry of the sensor to minimize the volume heat capacity while providing an electrical capacitance that is large compared to the stray capacitance in the circuit and the cryostat.

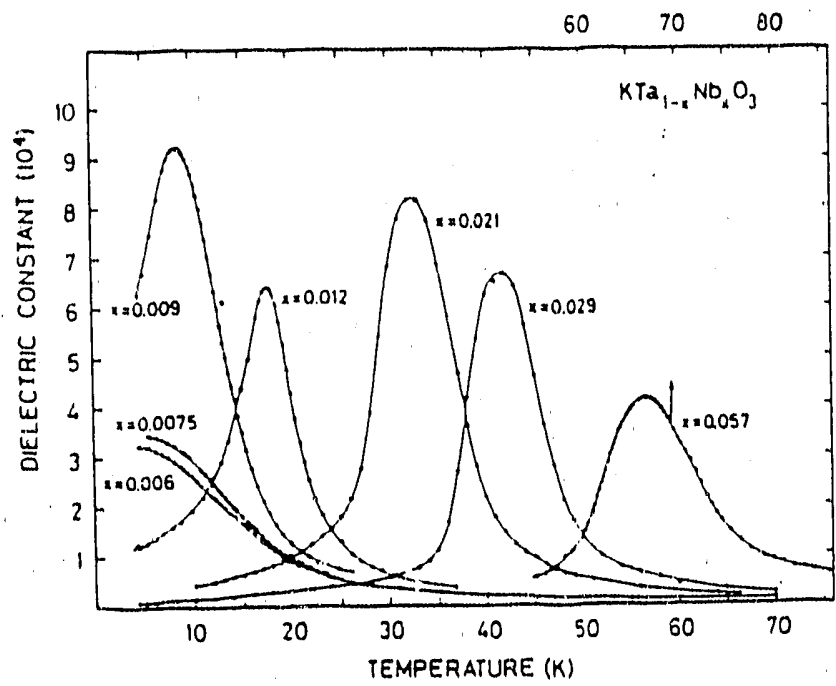

Figure 3. The temperature dependence of the dielectric constant for KTN as a function of the concentration, $x$. This data was reproduced from Rytz et al., $1983 .{ }^{4}$ 


\section{Table 1. Detector Geometry}

\begin{tabular}{cccc} 
Device & KTN Size & Absorber Size \\
& Are & Thickness & \\
\hline A & $2.85 \mathrm{~mm} \times 5.2 \mathrm{~mm}$ & $1.4 \mathrm{~mm}$ & None \\
B & $1 \mathrm{~mm} \times 1 \mathrm{~mm}$ & $100 \mu \mathrm{m}$ & $\begin{array}{c}\text { None } \\
\text { C }\end{array}$ \\
D & $1 \mathrm{~mm} \times 1 \mathrm{~mm}$ & $100 \mu \mathrm{m}$ & $1 \mathrm{~mm}^{2} \times 25 \mu \mathrm{rn}$ \\
& $250 \mu \mathrm{m} \times 250 \mu \mathrm{m}$ & $279 \mu \mathrm{m}$ & $1 \mathrm{~mm}^{2} \times 25 \mu \mathrm{m}$ \\
\hline
\end{tabular}

Table 1 lists the geometry of each microcalorimeter. Each is a parallel plate capacitor with evaporated layers of aluminum serving as the metallic surfaces, except for Sample A which uses palladium. In all cases, the KTN is connected to the JFET preamplifier. Sample A is a bulk chip of KTN in a mount (see Figure 4) which clamps the sample to the cold plate to make measurements of the capacitance of the device as a function of temperature. Sample B, as shown in Figure 5, is monolithic calorimeter and is supported by the two $18 \mu \mathrm{m}$ diameter Al:Si wires that are wedge bonded to the surface. Sample $C$ and Sample D are composite microcalorimeters. The composite calorimeters have a 1.0 $\mathrm{mm}^{2} \times 25 \mu \mathrm{m}$ thick absorber of Sapphire which is bonded with silver epoxy to the KTN. Sample C is supported in the same way as Sample B and has the same dimensions but with the absorber rotated at a $45^{\circ}$ angle and bonded to the KTN as shown in Figure 6. Sample D is supported by two graphite fibers as seen in Figure 7 and the KTN is $250 \mu \mathrm{m}^{2}$ by $279 \mu \mathrm{m}$ thick. This sample has $4000 \AA$ of aluminum has been evaporated on one side and $400 \AA$ of aluminum on the other.

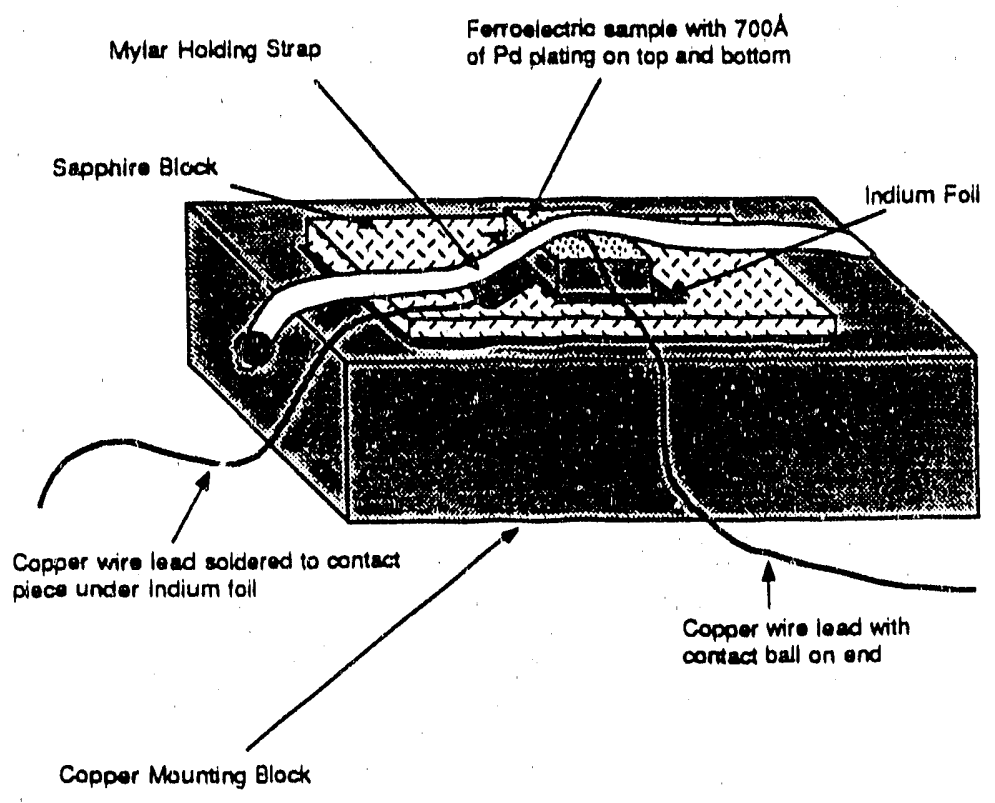

Figure 4. The mounting fixture, Sample A, used for measuring the dielectric constant and parallel resistance of the ferroelectric samples. 


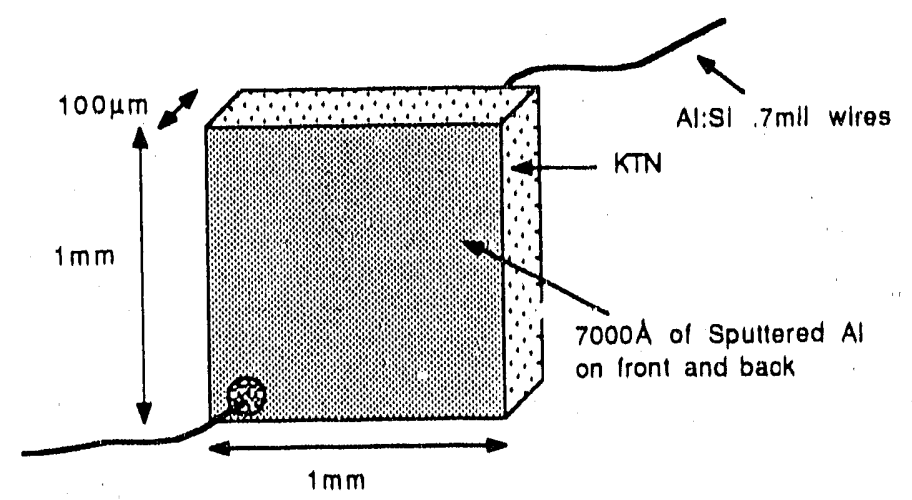

Figure 5. A schematic of Sample B

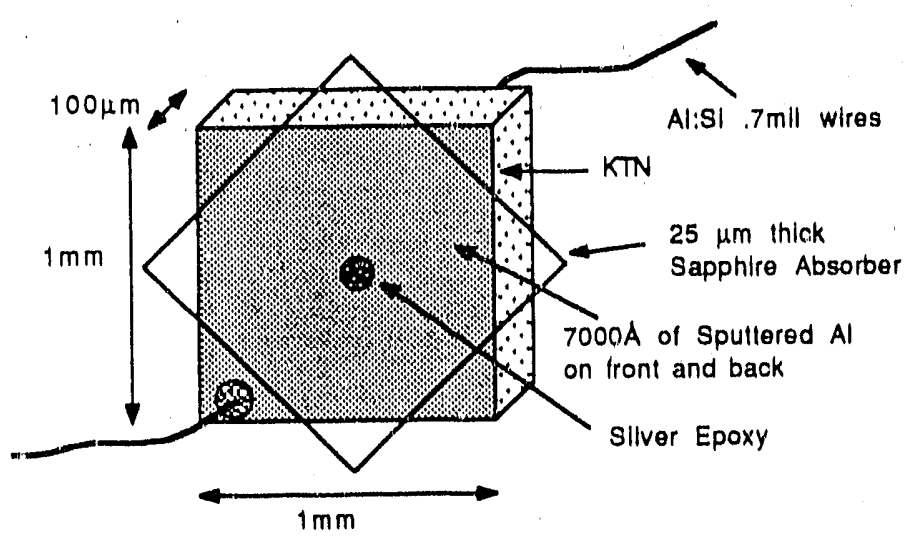

Figure 6. A schematic of Sample C

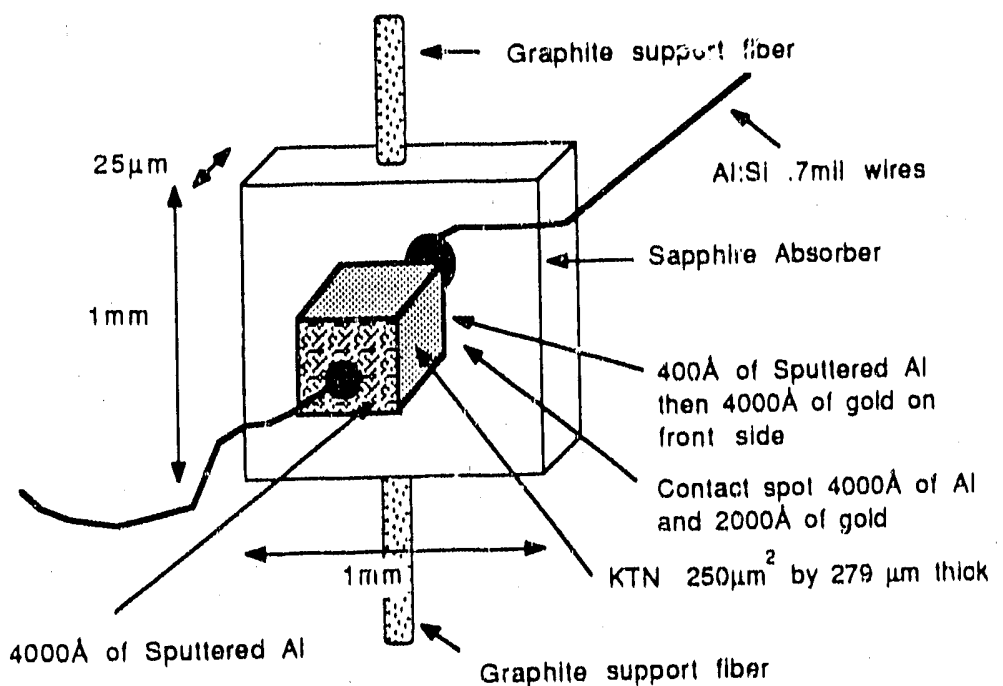

Figure 7. A schematic of Sample D 


\subsection{Dielectric constant measurements}

The charge sensitive preamplifier can be used to measure the capacitance and leakage resistance of these KTN devices. To measure the capacitance of the calorimeter we introduce a current, $I_{C}$, in the dielectric, by injecting a voltage step, $V_{i n}$, through the capacitor $C_{\text {bypass. }}$. Since $C_{\text {bypass }}$ is at least $10^{4}$ times as great as $C_{C}$, the voltage step is imparted predominantly across the dielectric $C_{c} \cdot I_{C}$ is then

$$
I_{c}=C_{c} \frac{d V_{\text {in }}}{d t}=\frac{d Q}{d t} .
$$

As discussed in Section 2.2 this current is integrated by the preamplifier feedback circuit to produce the output voltage $V_{\text {out }}$,

$$
V_{\text {out }}(t)=\int_{0}^{t} \frac{I_{c} d t}{C_{f}}=\frac{C_{C} V_{\text {in }}}{C_{f}} .
$$

The capacitance, $C_{c}$, is derived from the ratio of $V_{\text {out }}, V_{\text {in }}$ and the known feedback capacitance, $C_{f}$. It is

$$
C_{c}=\frac{V_{\text {out }}}{V_{\text {in }}} C_{f} .
$$

We note that the measurements were made by cooling the devices to the desired temperature with zero voltage bias across the KTN sample. The bias was then applied and the capacitance measured. A final measurement was then taken with zero bias to measure the hysteresis of the ferroelectric. As expected in ferroelectrics, the final capacitance was lower than the first value obtained with zero bias. To reproduce the initial zero bias measurement, the device must be warmed up past the ferroelectric transition temperature, $T_{c}$ (for $x=0.012 T_{c}=18 \mathrm{~K}$ ), and cooled again.

Capacitance measurements were made at several temperatures and as a function of voltage across the capacitor. In Figure 8 , the dielectric constant vs. temperature is plotted for the case with zero applied bias voltage. The curves have the same general shape as given by $\mathrm{Rytz}^{5}$ in Figure 3. The dielectric constant rises with falling temperature until the transition temperature $T_{c}$ is reached. Below this temperature, the dielectric constant drops quickly as the temperature falls.

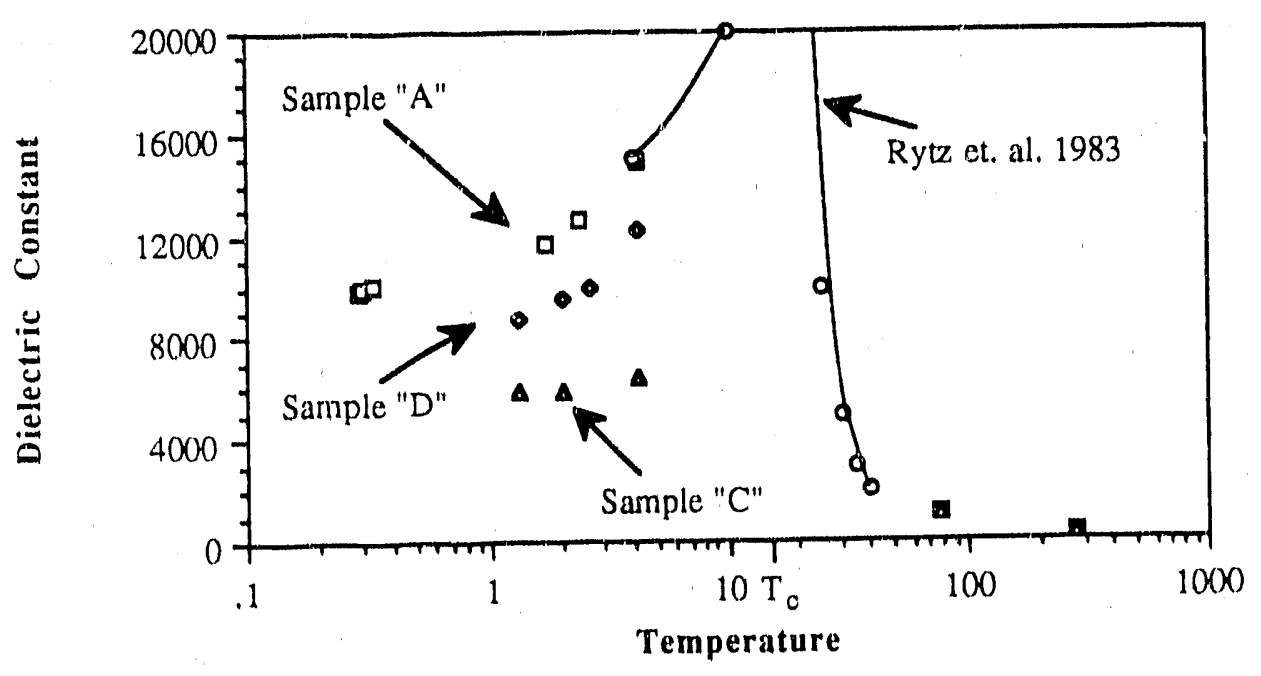

Figure 8. Measurements of the dielectric constant vs temperature with zero volts of bias.

According to those data the thickness has an effect on the dielectric constant as is shown in Figure 8. Sample A has a thickness of $1.4 \mathrm{~mm}$ as compared with Sample D which has a thickness of $279 \mu \mathrm{m}$ and Sample C with a thickness of 
$100 \mu \mathrm{m}$. The graph shows that as the samples become thinner they behave less like the bulk KTN; the value of the dielectric constant decreases and changes more slowly with temperature.

Figure 9 shows how the dielectric constant decreases as the applied voltage is increased. As the bias voltage is increased the change in dielectric constant with temperature decreases. This is because the domains in the crystals of KTN align with increasing field, and the average volume polarization of the material approaches an asymptotic value. Both the electric field and the thickness put constraints on the optimum geometry of the device. The hysteresis property is also evirlent; the value of $\varepsilon$ for the initial zero bias case is larger than the value reached by reducing the bias from $8(x) \vee$ to zeru volts.

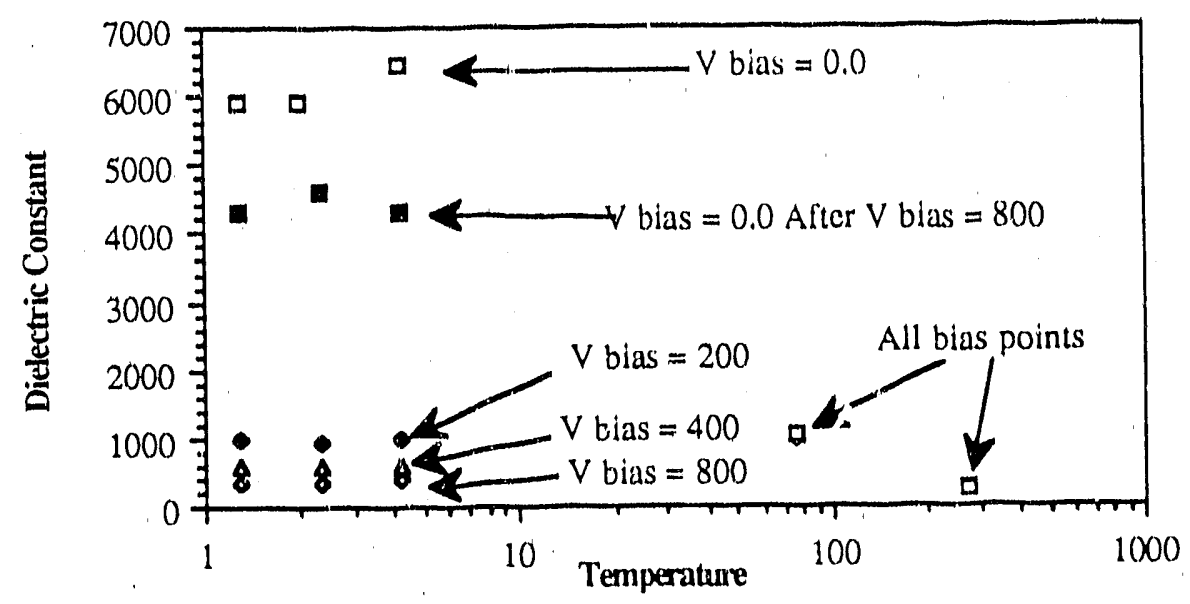

Figure 9. Measurement of the dielectric constant vs, temperature of sample " $B$ " with different bias voltages across the sample.

\subsection{Using KTN as a thermal sensor to detect IR and $6 \mathrm{MeV}$ alpha particles}

\subsubsection{Experimental set-up}

The KTN, in principle, could be used to fabricate a monolithic or composite calorimeter. We note that an electrical signal that does not have a thermal origin is observed when an energetic alpha particle interacts directly with a sample of KTN that has been cooled and has a voltage across it. We do not exactly know what underlying process causes this signal but we characterize some of the properties associated with this operating condition in Section 4.

The signal from a monolithic device may also have a thermal component, but we could not observe it using a monolithic geometry because of the non-thermal signals. To best study the KTN as a thermal sensor we have made a cornposite calorimeter Sample D, as shown in Figure 7. The KTN is attached to a larger sapphire absorber. The sapphire shields the KTN from any direct IR LED light or alpha particles. The capacitance was $6.1 \mathrm{pF}$ at 100 volts of bias at temperature of $1.3 \mathrm{~K}$. A $5.4 \mathrm{MeV}$ alpha particle source, ${ }^{241} \mathrm{Am}$, and an infrared LED light source were placed in the cryostalt in view of the detector. In the following measurements we cooled the detector through the ferroelectric transition temperature without applying a bias "oltage. The bias voltage was then applied at the low operating temperature.

\subsubsection{LED pulses}

Figure 10 shows the preamplifier output due to a LED flash lasting $10 \mu \mathrm{s}$ and with 100 volts detector bias. The risctime is $\approx 50 \mu \mathrm{s}$ and the decay is $\approx 600 \mu \mathrm{s}$. The size of the thernal output pulse scaled with input voltage as one would expect from the discussion in Section 2.2. From the thermal decay time of $\tau=600 \mu \mathrm{s}$ and the estimate of the thermal conductance of the aluminum wires, $G=10^{-6} \mathrm{~W} / \mathrm{K}$, we can estimate the heat capacity $C_{\mathrm{v}}=G \tau \approx 6 \times 10^{-10} \mathrm{~J} / \mathrm{K}$. 


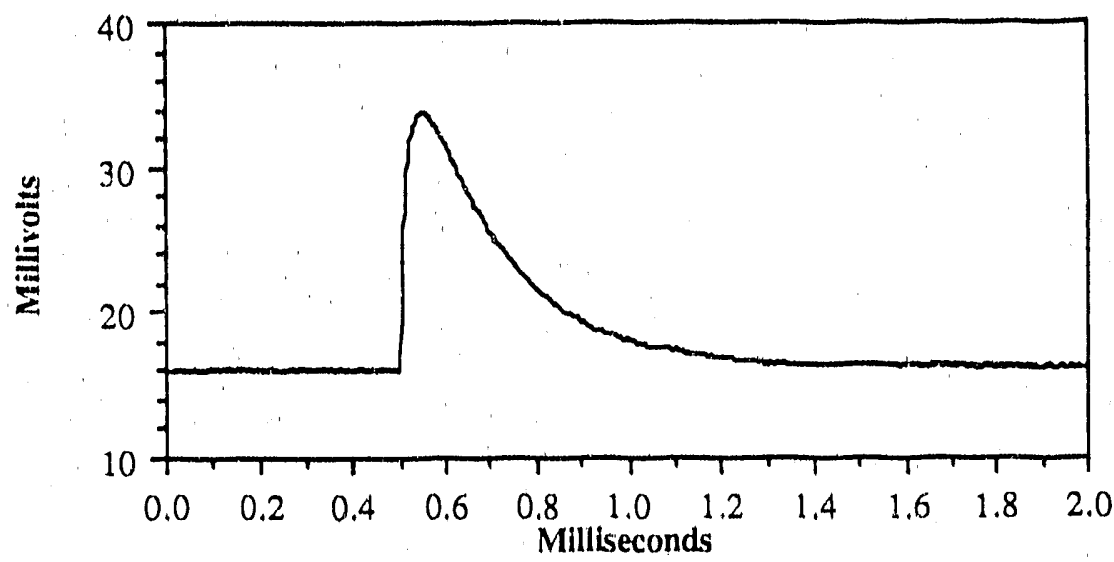

Figure 10. The response of a dielectric calorimeter at $1.3 \mathrm{~K}$ to a $10 \mu \mathrm{s}$ wide infrared LED pulse with a detector bias voltage of $100 \mathrm{~V}$.

\subsubsection{Alpha particle detection}

Figure 11 shows a typical signal produced by a $5.4 \mathrm{MeV}^{241} \mathrm{Am}$ alpha particle at the output of the post-amplifier which filters the broadband preamplifier signal and noise with a peaking time of $400 \mu \mathrm{s}$. Although the signal strength depends linearly on $V_{\text {bias }}$ (see Equation 2), it appears that the reduction in the term $\frac{1}{\varepsilon} \frac{d \varepsilon}{d T}$ has a stronger voltage dependence, causing the signal to noise ratio to peak with 100 volts of bias across the dielectric.

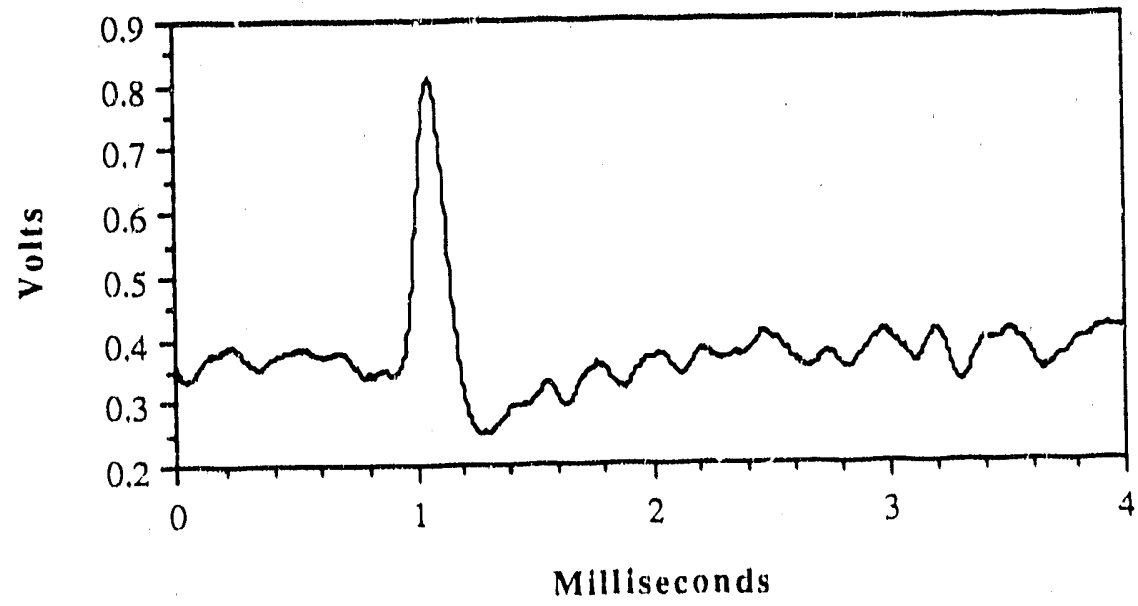

Figure 11. Thermal detection of an alpha particie with a dielectric culorimeter at $1.3 \mathrm{~K}$.. The signal has been filtered with $400 \mu$ s shaping time.

Figure 12 shows a spectrum of the ${ }^{241} \mathrm{Am}$ source. This source has two lines, one at $5.486 \mathrm{MeV}$ with a $85 \%$ branching ratio and the other at $5.443 \mathrm{MeV}$ with a $12.8 \%$ branching ratio. Our current resolution of about $0.2 \mathrm{MeV}$ is not good enough to resolve these two lines. This spectrum was taken with 100 volts of bias and a pest amplifier shaping tume of $100 \mu \mathrm{s}$. We attribute the relatively poor signal to noise to the value of $\frac{1}{\varepsilon} \frac{d \varepsilon}{d T}$, which is smaller than expected, due to the high electric field. 


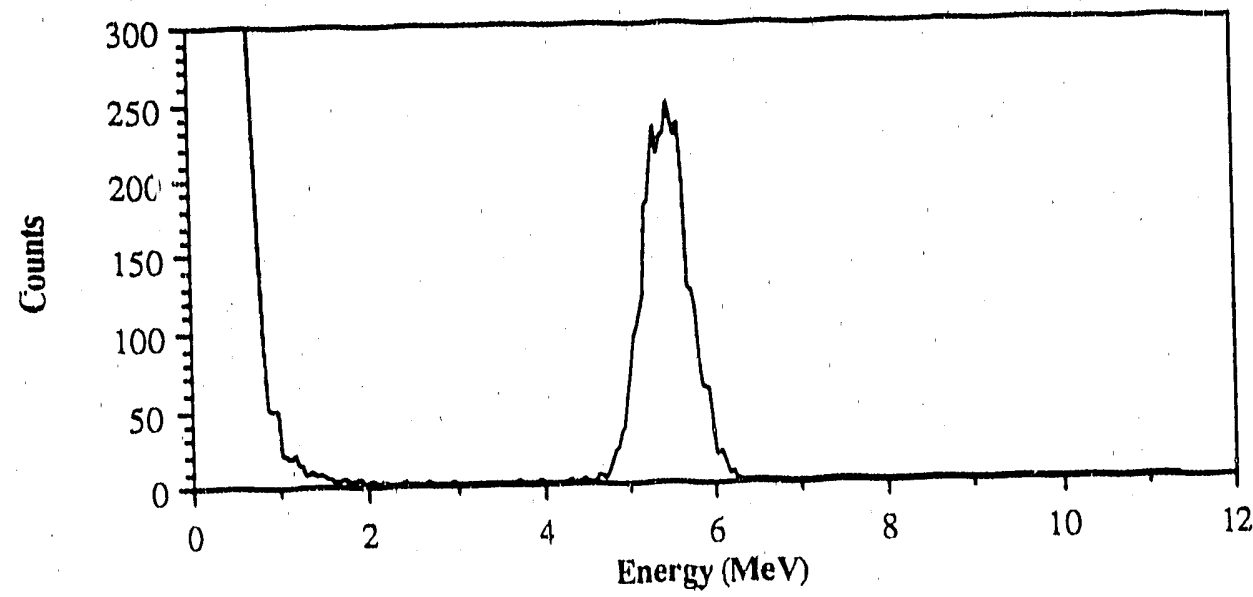

Figure 12. A thermal ${ }^{241} \mathrm{Am}$ alpha spectrum taken at $1.3 \mathrm{~K}$ with 100 volts of bias and a post amplifier shaping time of $100 \mu \mathrm{s}$.

\section{NON-THERMAL ALPHA DETECTION}

As mentioned above, we have discovered that a monolithic KTN capacitor can detect individual $6 \mathrm{MeV}$ alpha particles by a mechanism that is clearly non-thermal. They are observed at room temperature and $77 \mathrm{~K}$ and produce pulse heights at the preamplifier output of a few millivolts. At these temperatures the pulse heights were independent of the bias voltage and were observed with bias voltages lower than 2 volts. Figure 13 shows an ${ }^{241} \mathrm{Am}$ spectrum taken at $77 \mathrm{~K}$ with $\approx 2$ volts of bias.

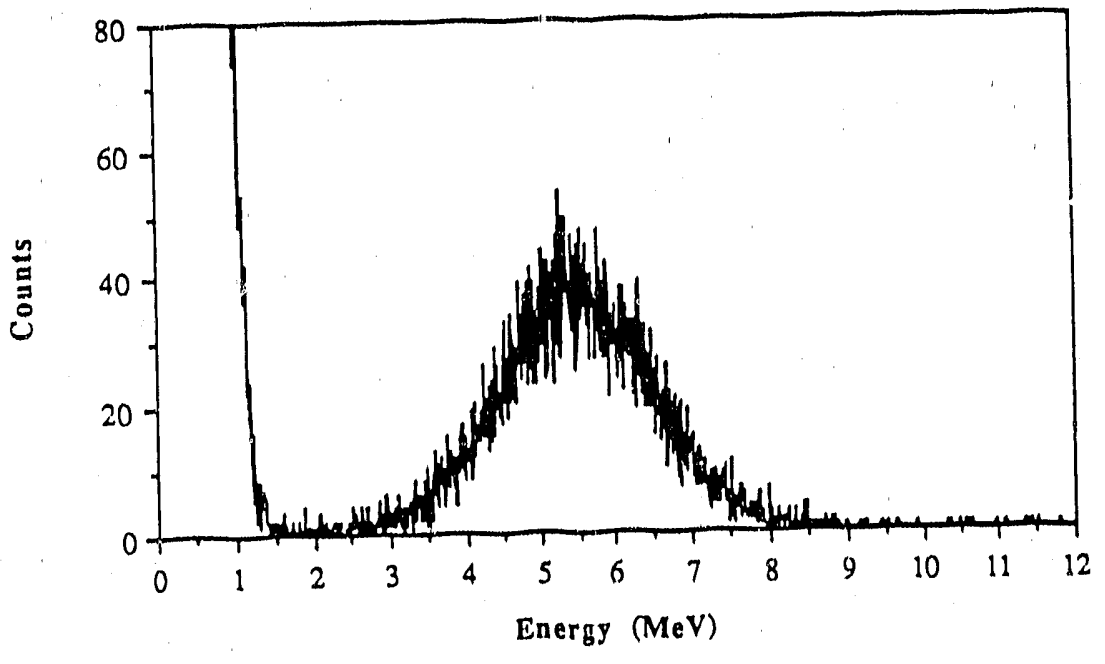

Figure 13. A non-thermal ${ }^{241} \mathrm{Am}$ alpha spectrum taken at $77 \mathrm{~K}$ with 2 volts of bius and a post amplifier shaping time of $1.2 \mathrm{~ms}$.

The pulses were also observed in the range between $4.2 \mathrm{~K}$ to $1.3 \mathrm{~K}$. Figure 14 shows an example of a $6 \mathrm{McV}$ alpha pulse as seen at the preamplifier output. The amplitudes of these pulses are 50 times larger than those observed at $77 \mathrm{~K}$ and more than a factor of 100 times larger than the thermal pulses discussed in Section 3. Figure 15 shows the pulse at the output of the post amplifier with a shaping time of $3.6 \mathrm{~ms}$. By comparing Figures 15 and Figure 11, one can see the improvement in the signal to noise ratio of the non-thermal pulses compared to that of the thermal pulses. 


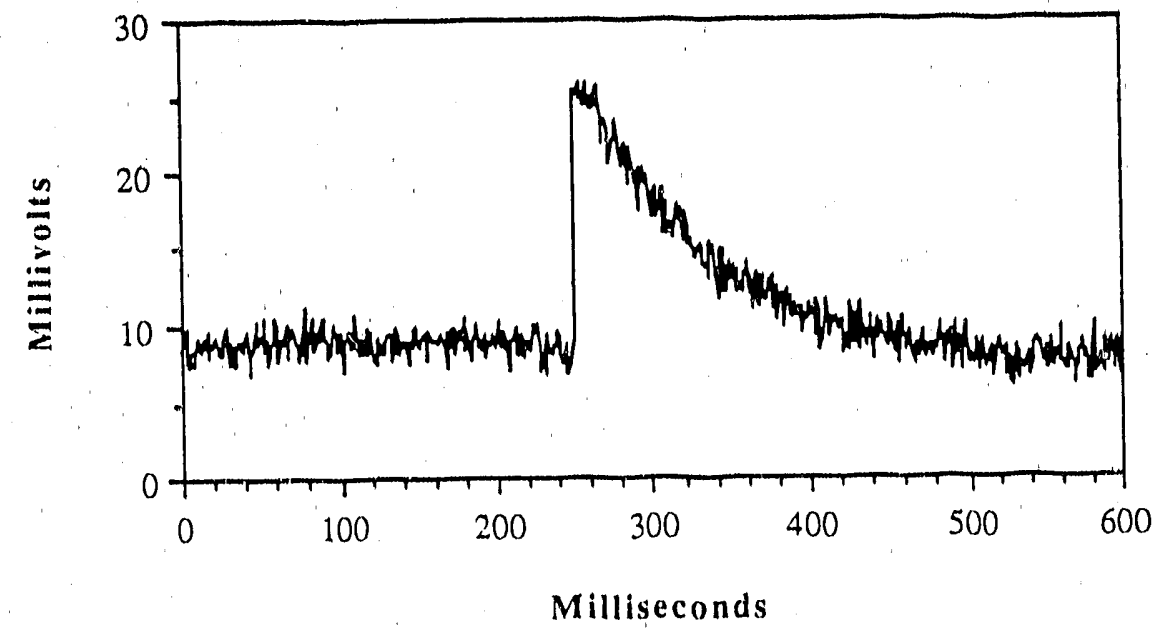

Figure 14. A typical non-thermal $6 \mathrm{MeV}$ alpha pulse measurement at $1.3 \mathrm{~K}$ as seen out of the preamplifier with 800 volts of bias,

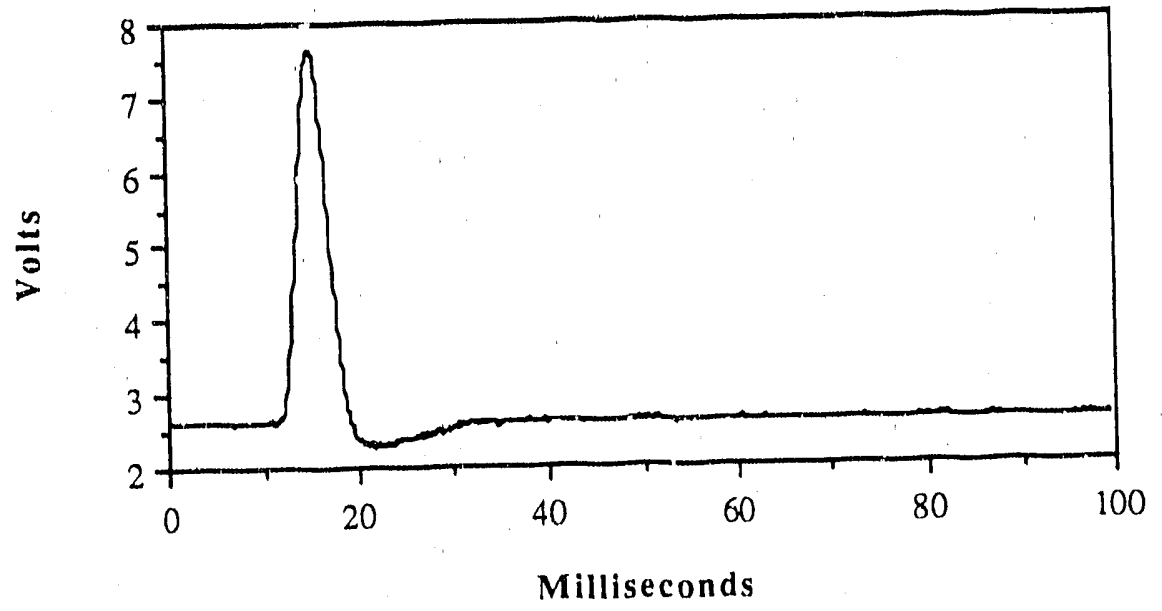

Figure 15. A non-thermal alpha pulse measured at $1.3 \mathrm{~K}$ as viewed out of the post amplifier with a shaping time of $3.6 \mathrm{~ms}$.

We know that these signals are not a signature of a temperature change in the dielectric because:

(a) The signal count rate dropped when sapphire, a known $I R$ and alpha particle absorber was attached to the front of the KTN. In Sample $C$ the count rate dropped by a factor of $\approx 6$ which corresponded to the drop in exposed area of KTN. In Sample D, where the KTN was hidden from the alphas by a sapphire absorber, the count rate dropped to zero.

(b) Since the sign of $\mathrm{d} \varepsilon / \mathrm{dT}$ changes at the ferroelectric transition point, (see Figure 3), a thermally produced signal would also change sign when the temperature crosses this point. No such change in sign of the signals was observed.

(c) The device has a higher heat capacity at higher temperature. We would not expect the thermal signal to be detectable at $77 \mathrm{~K}$ and room temperature. Signals detected in these temperature ranges.

(d) The decay time of the electrical signal, 0.5 to $1.0 \mathrm{~s}$, is characteristic of the preamplifier feedback time constant rather than the $400 \mu \mathrm{s}$ decay time observed when the device is operating in a thermal mode. 
We have obtained signal to noise ratios of 2000 to $4000: 1$ with $6 \mathrm{MeV}$ alpha particles at $1.3 \mathrm{~K}$ with a bias of 930 volts. The pulses between $4.2 \mathrm{~K}$ and $1.3 \mathrm{~K}$ did scale linearly with the applied bias voltage and disappeared at zero bias. The electronic noise at these operating conditions was better than $\Delta \mathrm{E}_{(\mathrm{FWHM})} \approx 3 \mathrm{KeV}$ calibrated against $6 \mathrm{MeV}$ alpha particles. Although the signal to noise is excellent, the resolution is relatively poor as is seen in Figure 15 which shows the spectrum of a multi-nuclide $228 \mathrm{Th}$ alpha source. This spreading could be due to a position sensitive effect in the energy to voltage conversion process in the KTN.

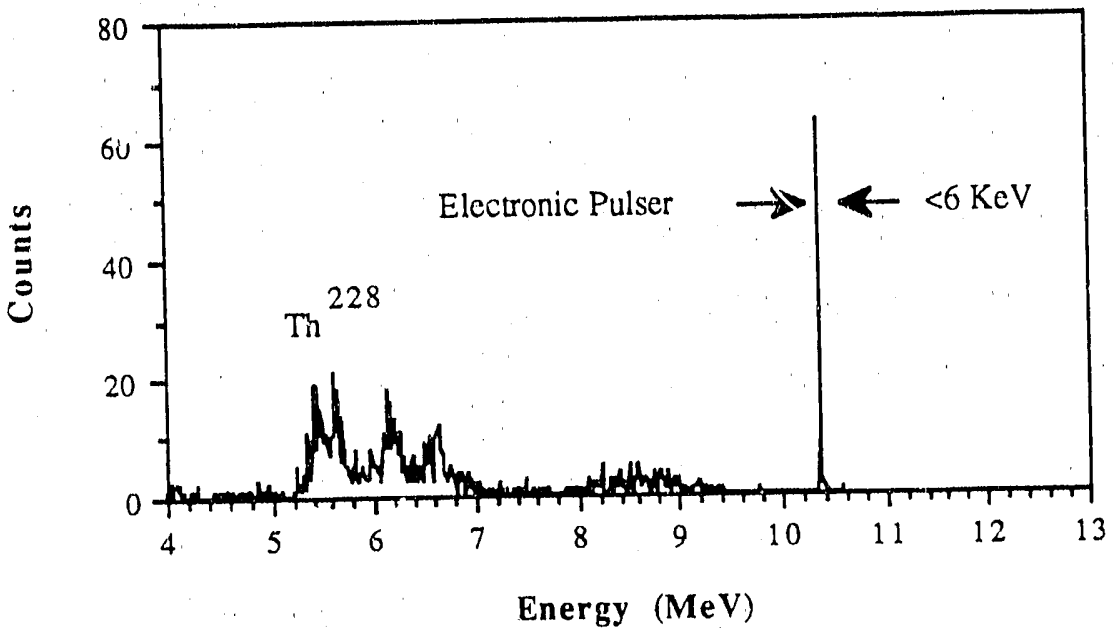

Figure 16. A non-thermal ${ }^{228} \mathrm{Th}$ alpha spectrum taken at $1.3 \mathrm{~K}$ with 930 volts of bias and a post amplifier shaping time of $3.6 \mathrm{~ms}$.

\section{FUTURE EXPERIMENTS}

Future plans include optimizing the design of the thermal detectors by adjusting the size of the detector and absorber as well as testing crystals doped with less niobium, such as the fraction, $x=0.009$. We will also investigate other ferroelectric crystals such as $\mathrm{SrCax}_{\mathrm{Ti}} \mathrm{x}_{\mathrm{x}} \mathrm{O}_{3}$ and $\mathrm{SrBax}_{\mathrm{T}} \mathrm{Ti}_{1-\mathrm{x}} \mathrm{O}_{3}$ which have the potential of giving even higher dielectric constants. ${ }^{7}$ We will also explore the effects of applying bias while cooling the KTN through the transition point. Further studies will be made of the non-thermal pulses to determine the process responsible for these pulses and to determine possible applications.

\section{ACKNOWLEDGEMENTS}

We wish to thank Dan Rytz of the Sandoz corporation for supplying the $\mathrm{KTa}_{1-\mathrm{x}} \mathrm{Nb}_{\mathrm{x}} \mathrm{O}_{3}$ samples used here. This work is supported by NASA grant NAGW-1686 and performed under the auspices of the U.S. Department of Energy by Lawrence Livermore National Laboratory under contract No. W-7405-ENG-48.

\section{REFERENCES}

1. Labov, S., Silve:, E., et.al., "X-ray Microcalorimeter with Germanium Resistance Thermometers"' in Proc. SPIE 1344,1990 (this volume).

2. Silver, E.H., Labov, S.E., Goulding, F., Madden, N., Landis ,D., and Beeman, J., "A New Microcalorimeter Concept for Photon Counting X-ray Spectroscopy", Nucl. Instr. and Methods, A 277, pp. 657-663, 1989. 
3. Goulding, F.S., "Pulse-Shaping in Low-Noise Nuclear Amplifiers: A Physical Approach to Noise Analysis", Nucl. Instr. and Meth., 100, p. 493, 1972.

4. Silver, E.H., Labov, S.,Pfafman, T., Goulding, F., Madden, N., Landis, D., and Beeman, J., "A New Thermal Sensor for X-ray Microcalorimetry", in Proc. of NASA Workshop on X-ray Astronomy in the 21st Century, Taos, N.M., Dec. 1989.

5. Rytz, D., Chatelain, A., and Hochli, U.T., "Elastic Properties in Quantum Ferroelectric $\mathrm{KTa}_{1-\mathrm{x}} \mathrm{Nb}_{\mathrm{x}} \mathrm{O}_{3}$ ", Phys. Rev. $B, 27,11$, p.p. 6830 , (1983).

6. Lawless, W.N. et al., "Specific Heat Measurements in $\mathrm{KTa}_{1-\mathrm{x}} \mathrm{Nb}_{\mathrm{x}} \mathrm{O}_{3}$ Quantum Ferroelectricity and Glasslike Properties", in Proceedir "So the Sixth International Meeting on Ferroelectricity, Kobe, Jap. J. Appl. Phys., 24 ,

7. Bednorz, J.G., Muller, K.A., " $\mathrm{Sr}_{1-\mathrm{x}} \mathrm{Ca}_{\mathrm{x}} \mathrm{TiO}_{3}$ : An XY Quantum Ferroelectric with Transition to Randomness",

Phys. Rev. Lett., 52, pp. 2289-2292, (1984). 

